\title{
Dynamical partitioning of directional ocean wave spectra
}

\author{
Pierre Ailliot $^{\mathrm{a}, *}$, Christophe Maisondieu ${ }^{\mathrm{b}}$, Valérie Monbet ${ }^{\mathrm{c}}$
}

\author{
a Université de Brest, France \\ ${ }^{\mathrm{b}}$ Ifremer, France \\ ${ }^{\mathrm{c}}$ Université de Rennes, France \\ *: Corresponding author : Pierre Ailliot, Tel.: +33 298016203 ; email address : ailliot@univ-brest.fr
}

\begin{abstract}
:
Directional wave spectra generally exhibit several peaks due to the coexistence of wind sea generated by local wind conditions and swells originating from distant weather systems. This paper proposes a new algorithm for partitioning such spectra and retrieving the various systems which compose a complex seastate. It is based on a sequential Monte-Carlo algorithm which allows to follow the time evolution of the various systems. The proposed methodology is validated on both synthetic and real spectra and the results are compared with a method commonly used in the literature.
\end{abstract}

Keywords: Partitioning algorithm ; Directional wave spectrum ; Sequential Monte Carlo algorithm

\section{Introduction}

For a wide range of marine activities (structural design, marine energy exploitation, forecasting, climate assessment, etc), an accurate description of the sea states, or more generally of wave climate, is crucial. The waves existing at a specific location are generally classified as wind sea when they are generated locally by the wind or swell when they are radiated from distant wind systems. The resulting sea state can be described by a spectrum which provides information about the energy transmitted by all existing wave systems. Figure 1 shows an example of series of directional spectra considered in this work. This set of spectra show the time evolution of the distribution of wave energy as a function of the frequency and direction at a fixed location. Such spectra are not easy to handle (nor to store) and it is often more convenient to extract synthetic parameters which characterize their main features. During the last decades, several partitioning algorithms have been proposed in order to identify the wave systems as well as their features from time series of directional wave spectra.

Most partitioning methods [4], [3] are based on Gerling's [1] and Hasselman's ([7], [6]) works and consist in a three steps algorithm. In the first step, the spectrum is separated in several areas, each of them being associated with a spectral energy peak. In [1], a time-consuming recursive algorithm, which assigns each spectrum value to a path of steepest ascent associated with a local peak, is used. All paths leading to the same peak are assigned to a distinct spectral partition. In more recent works ([5], [16]), this step is improved using efficient image processing routines developed in topographic imagery for making watershed delineations ([17], [18]). Although this approach is now used in most of the partitioning algorithms, some authors ([2], [11]) propose an alternative method to identify the most important energy peaks of a spectrum; they first locate the peaks within the spectrum looking for the global maximum, then associate each peak to an area delimited around it and the process is repeated for the next maximum, etc. (see for instance [20] for a comparison of the two methods). Once the partition is obtained, each area is associated with a wave system which can be characterized by synthetic parameters such as the corresponding significant wave height, the position of the peak in frequency and direction and sometimes also some frequential and directional spreading parameters. Unrealistic wave systems, associated for example with noise in the spectrum, are sometimes identified. Specific procedures allow merging these 


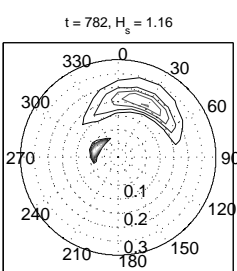

$\mathrm{t}=806, \mathrm{H}=0.99$
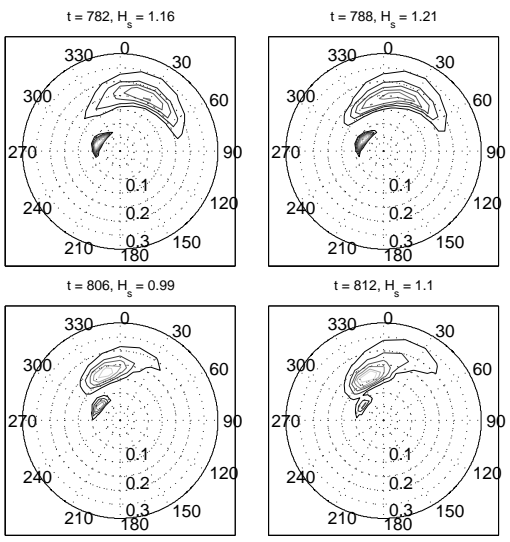

$\mathrm{t}=812, \mathrm{H}_{\mathrm{s}}=1.1$
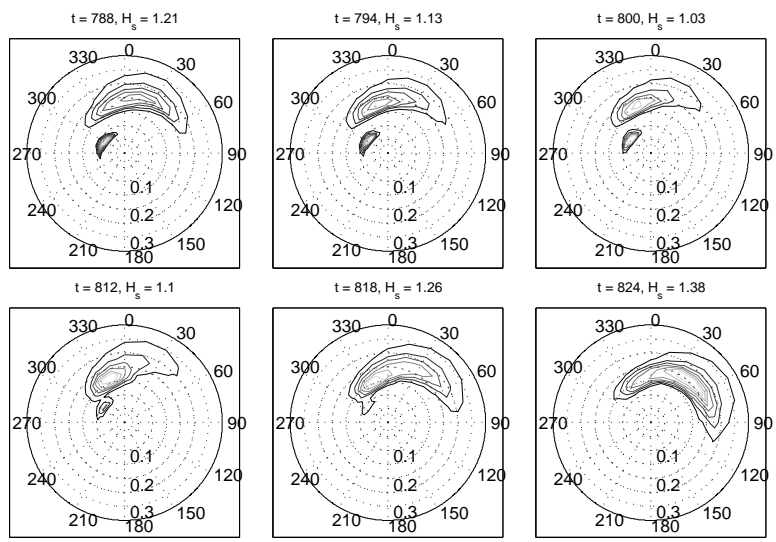

$\mathrm{t}=818, \mathrm{H}_{\mathrm{s}}=1.26$
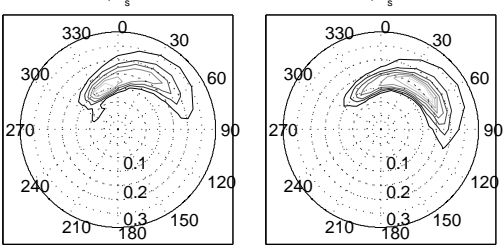

Figure 1: Time series of WWIII spectra (6 hours between successive spectra). The time given in the titles corresponds to the number of hours since the beginning of 2009 .

artifacts with the identified physical wave systems. The following step of the partitioning process consists in identifying wind sea and swell systems. First, the wind sea is identified using local wave age criteria as in [19]. This approach has also been implemented in [14] where specific cases for which the method may fail to correctly separate wind sea and swell are highlighted. This problem is further discussed and improved in [8]. Then swell systems with close peaks are merged and the areas with low total energy are negelected. Some algorithms, such as the Spectral Partitioning for Operational Parameters Identification (denoted SPOP in the sequel) algorithm (see [10]), perform an additional step where specific parametric spectrum models are fitted in order to improve the values of the parameters associated to each system and to insure that the total energy of the resulting spectrum is equal to the one of the reference (observed) spectrum. The last step is a clustering task which allows to track the systems in time and link together the wave systems identified at successive time steps. Cross-assignment is usually performed on the system parameters using classical clustering algorithms.

Most of the partitioning methods proposed in the literature are very similar and their differences mostly lie in the tuning of some of the algorithm's parameters such as, for instance, the definition of the closeness of two swell peaks. A limitation of the watershed delineations approach is that it may fail to separate systems with close frequencies and directions. For instance, Figure 2 shows the spectrum obtained when mixing two Jownswap spectra which parameters correspond respectively to typical values for swell and wind sea systems. The resulting spectrum exhibits only one local maximum and thus standard partitioning methods will not be able to retrieve the two systems except if the directional distribution provides extra-information. Figure 1 provides another example of such situation. The first spectra in the sequence clearly exhibit two well separated wave systems: a swell propagating from the west-north-west and a wind sea propagating from the north with wider directional and spectral spreading and with higher peak frequency. The distance between the two peaks decreases progressively so that the last spectrum has only one local maximum although it is likely that the two wave systems are still present. In such situation, using the partition obtained at the previous time step may help retrieving the two wave systems. In the methodology introduced in this paper, this is done by assuming that the synthetic spectral parameters associated to the various wave systems follow simple stochastic models. Since these spectral parameters are not observable, they are introduced as a hidden process and the sequence of observed spectra is related to the hidden sequence using standard parametric models for wave spectrum. The tracking of the various wave systems becomes a natural output of the algorithm such that no post treatment is required; this is another expected advantage of our methodology over watershed delineations approaches.

The performance of the algorithm is illustrated using a time series of wave spectra in the North East Atlantic $(5 \mathrm{~W}, 45 \mathrm{~N})$ retrieved from the wind-wave model WWIII run by the ARGOSS company. The time series covers the period from January to March 2009 with a spectrum every hour. A short part of the sequence is shown on Figure 1.

The partitioning algorithm is described in Section 2. Then in Section 3, the stochastic models used to 


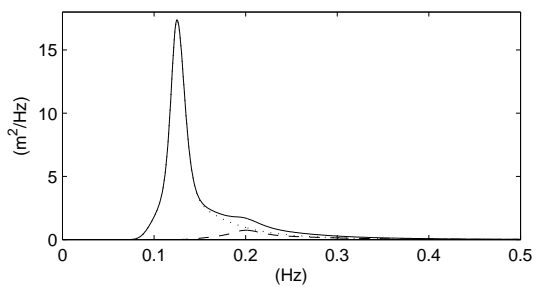

Figure 2: Spectrum (solid line) obtained by mixing a JONSWAP spectrum with parameters $H_{s}=3 \mathrm{~m}, T_{p}=8 \mathrm{~s}$ and $\gamma=5$ (dotted line) and a JONSWAP spectrum with parameters $H_{s}=1 \mathrm{~m}, T_{p}=5 \mathrm{~s}$ and $\gamma=2$ (dashed line).

describe the time evolution of the wave systems are detailed. In Section 4, numerical results are first presented for a synthetic sequence of spectra. Then the results obtained on the WWIII spectra are discussed and compared to the results obtained using the SPOP algorithm. Conclusions and perspectives are given in Section 5 .

\section{Methodology}

A directional wave spectrum $S(\omega, \theta ; t)$ is a scalar function which provides information about the distribution of the wave energy as a function of the frequency $w$ and the direction $\theta$ at the observation time $t \in\{1, \ldots, T\}$. Many engineering studies rely on synthetic sea-state parameters which summarize the information contained in the directional wave spectra, such as the significant wave height $H_{s}$, the peak period $T_{p}$ and the mean wave direction $\theta_{m}$. Various parametric models have also been proposed in the literature for reconstructing directional spectra from the synthetic sea-state parameters (see e.g. [12]). Hereafter, we denote $S_{\text {par }}(\omega, \theta ; X)$ the directional spectrum obtained by multiplying a JONSWAP spectrum with shape parameter $\gamma$ for the frequency distribution and a $\cos ^{2 s}$ function for the angular distribution with $X=\left(H_{s}, T_{p}, \gamma, \theta_{m}, s\right)$. This parametric form has been chosen in this work because it is probably the most common one and it can accommodate a wide range of spectrum shapes but the method proposed hereafter can easily handle other parametric models.

Sea-states are generally composed of several superimposed wave systems and the usual unimodal parametric models such as the one introduced above are not appropriate in such cases Hereafter we thus assume that

$$
S(\omega, \theta ; t)=\sum_{k=1}^{K_{t}} S_{p a r}\left(\omega, \theta ; X^{(k)}(t)\right)+S_{r e s}(\omega, \theta ; t)
$$

with $K_{t}$ the number of wave systems at time $t$ and $X^{(k)}(t)=\left(H_{s}^{(k)}(t), T_{p}^{(k)}(t), \gamma^{(k)}(t), \theta_{m}^{(k)}(t), s^{(k)}(t)\right)$ the synthetic parameters associated with the system number $k$ at time $t$. $S_{\text {res }}(\omega, \theta ; t)$ represents the difference between the observed and the parametric spectra and may model the observation error but also the error due to a bad specification of the parametric model. Finally, we denote $X(t)=\left(X^{(1)}(t), \ldots, X^{\left(K_{t}\right)}(t)\right)$ and

$$
S_{\text {par }}(\omega, \theta ; X(t))=\sum_{k=1}^{K_{t}} S_{\text {par }}\left(\omega, \theta ; X^{(k)}(t)\right)
$$

the parametric spectrum associated to $X(t) .(X(1), \ldots X(T))$ is an unobserved time series of synthetic sea-sate parameters that we would like to retrieve from the sequence of observed spectra.

The algorithm proposed in this paper computes successive approximations of $X_{t}$ by cycling through the following steps.

Simulation of a large number of possible scenarios (also called particles in the sequel) at time $t$ coherent with the systems identified at the previous time step, i.e. $X_{t-1}$. In practice, we make the assumptions described hereafter. 
- Each of the $K_{t-1}$ systems present at time $t-1$ can persist, merge with another system or vanish at time $t$. When they persist, the parameters of the systems are assumed to evolve according to the simple stochastic models described in Section 3 which can be easily simulated.

- New swell and wind sea systems may appear between time $t-1$ and time $t$. The distributions used to generate the spectral parameters of the new systems are described in Section 3 .

In this work, we have limited the number of new wave systems to one of each type (i.e. one swell and one wind sea) at each time step but this assumption could easily be relaxed. This leads to a maximum number of $K_{t-1}+2$ wave systems, and each of these wave systems can actually be present or not at time $t$. We have $P_{t}=2^{K_{t-1}+2}$ possible combinations of wave systems which will be denoted $\mathcal{M}_{1}, \ldots, \mathcal{M}_{P_{t}}$. Step 1 of the algorithm (see Table 1) consists in listing these possibilities. In Step 2.a, $N$ possible values of the spectral parameters for each combination of wave systems are generated. These values are denoted $X_{i, \mathcal{M}_{p}}(t)$ for $i \in\{1, \ldots, N\}$ and $p \in\left\{1, \ldots, P_{t}\right\}$ and the stochastic models which are used to generate these $N P_{t}$ scenarios are described more precisely in Section 3.

Weighting of the scenarios simulated in the previous step (Step 2.b). The weights are linked to the distance between the observed spectrum and the spectrum associated to the simulated spectral parameters and are given by

$$
\pi_{i, \mathcal{M}_{p}}(t)=\phi\left(S(:, t) ; S_{i, \mathcal{M}_{p}}(:, t), \Omega\right)
$$

where $S(:, t)$ denotes a vector which contains the observed spectrum, $S_{i, \mathcal{M}_{p}}(:, t)$ the parametric spectrum reconstructed from $X_{i, \mathcal{M}_{p}}(t)$ at the same frequencies and directions than the observed spectrum and $\phi(. ; \mu, \Omega)$ the probability density function of a multivariate Gaussian distribution with mean $\mu$ and covariance matrix $\Omega$ (see Eq. (9) for more details). According to Eq. (1), the weight $\pi_{i, \mathcal{M}_{p}}(t)$ can be interpreted as the probability of observing $S(\omega, \theta ; t)$ if $X_{i, \mathcal{M}_{p}}(t)$ is the correct partition at time $t$ and $S_{\text {res }}(:, t)$ is a Gaussian vector with zero mean and covariance matrix $\Omega$. The choice of the Gaussian probability density function is usual in the statistical literature and in particular when working with particle filters. It allows the definition of a weight based on the usual Euclidean distance and associate larger weight to parametric spectra which are close to the observed ones.

Selection of the best combination of wave systems (Step 3). Different methods have been tested in order to chose the optimal combination of wave systems at time $t$, i.e. the best value $p_{0}(t) \in\left\{1, \ldots, P_{t}\right\}$. The first criterion was based on the following quantities

$$
\Pi_{\mathcal{M}_{p} \mid X}(t) \propto \Pi_{\mathcal{M}_{p}}(t) \Pi_{X \mid \mathcal{M}_{p}}(t)
$$

which aimed at estimating the posterior probability of the model $p \in\left\{1, \ldots, P_{t}\right\}$ given the data, following standard methods in Bayesian statistics (see e.g. [15]). In this expression, $\left(\Pi_{\mathcal{M}_{p}}(t)\right)_{p \in\left\{1, \ldots, P_{t}\right\}}$ is interpreted as a prior distribution on the set of possible combinations of wave systems at time $t$ and $\Pi_{X \mid \mathcal{M}_{p}}(t)$ is defined as the mean weight

$$
\Pi_{X \mid \mathcal{M}_{p}}(t)=\frac{1}{N} \sum_{i=1}^{N} \pi_{i, \mathcal{M}_{p}}(t)
$$

and may be interpreted as the likelihood of the observed spectrum if $p$ is the correct combination of wave systems at time $t$. In practice, we obtained better results when replacing the mean by a quantile in order to filter out the less likely values of $X_{i, \mathcal{M}_{p}}(t)$. In the following, $\Pi_{X \mid \mathcal{M}_{p}}(t)$ is defined as the empirical quantile of $\left(p_{i, \mathcal{M}_{p}}(t)\right)_{i \in\{1, \ldots, N\}}$ associated to the probability $q$ and $p_{0}(t)$ as the value of $p$ which maximizes $\left(\Pi_{\mathcal{M}_{p} \mid X}(t)\right)_{p \in\left\{1, \ldots, P_{t}\right\}}$ where $\Pi_{\mathcal{M}_{p} \mid X}(t)$ is defined by Eq. (4). In practice, the choice of the prior distribution permits to favor or penalize some combinations of wave systems. This is further discussed in Section 4.

Reestimation of the spectral parameters (Step 4). The previous step leads to an estimate of the best combination of wave systems $p_{0}(t)$ together with a set of weighted particles $\left(X_{i, \mathcal{M}_{p_{0}(t)}}(t), \pi_{i, \mathcal{M}_{p_{0}(t)}}(t)\right)$ from which we retrieve a first estimate of $X_{t}$ by taking the weighted mean over the more likely particles

$$
X_{t}^{(0)}=\frac{1}{\sum_{i \in A} \pi_{i, \mathcal{M}_{p_{0}(t)}}(t)} \sum_{i \in A} \pi_{i, \mathcal{M}_{p_{0}(t)}}(t) X_{i, \mathcal{M}_{p_{0}(t)}}(t)
$$


where $A$ represents the set of the $100(1-q)$ percent highest values of $\pi_{i, \mathcal{M}_{p_{0}(t)}}(t)$. The directional spectrum associated to $X_{t}^{(0)}$ is generally close to the observed spectrum but we refine the value by adding a numerical optimization step. The function that we minimize, defined as

$$
\phi\left(S(:, t) ; S_{\text {par }}\left(:, X_{t}\right), \Omega\right)
$$

is a distance between the observed and the parametric spectra. We run the optimization algorithm starting from $X_{t}^{(0)}$. This leads to an updated value of the spectral parameters which is close to $X_{t}^{(0)}$ but fits better to the observed spectrum. The systems with very low energy (significant wave height below 0.1 meter) are removed before going to the next step.

The different steps of the algorithm are summarized in Table 1 . At time $t$, Step 1 consists in listing all the possible combinations of sea state systems given the combination selected at $t-1$. In Step 2, a large number of particles is simulated for each combination and weights are associated to the particles. In Step 3 the weights, which can be interpreted as the likelihoods of the observed spectrum if the particles give the correct partition, are used to find the best combination. Finally, Step 4 consists in a numerical optimization step which permits to improve the parameters of the selected combination in order to better match with the observed spectrum.

$$
\begin{aligned}
& \text { Step } 1 \\
& \text { List of possible } \\
& \text { combinations } \\
& \text { a. Simulation of the particles } \\
& \text { b. Computation of weights } \\
& \begin{array}{c}
\text { Step } \mathbf{3} \\
\text { Selection of the best }
\end{array} \\
& \text { combination } \\
& \text { Step } 4 \\
& \left(\mathcal{M}_{1}, \Pi_{\mathcal{M}_{1}}(t)\right) \rightarrow\left\{\begin{array}{l}
\left(X_{1, \mathcal{M}_{1}}(t), \pi_{1, \mathcal{M}_{1}}(t)\right) \\
\vdots \\
\left(X_{q N, \mathcal{M}_{1}}(t), \pi_{q N, \mathcal{M}_{1}}(t)\right) \\
\vdots \\
\left(X_{N, \mathcal{M}_{1}}(t), \pi_{N, \mathcal{M}_{1}}(t)\right)
\end{array}\right. \\
& \begin{array}{ccc} 
& \nearrow & \\
& & \vdots \\
& & \\
& \searrow &
\end{array} \\
& \left(\mathcal{M}_{P_{t}}, \Pi_{\mathcal{M}_{P_{t}}}(t)\right) \rightarrow\left\{\begin{array}{l}
\left(X_{1, \mathcal{M}_{P_{t}}}(t), \pi_{1, \mathcal{M}_{P_{t}}}(t)\right) \\
\vdots \\
\left(X_{q N, \mathcal{M}_{P_{t}}}(t), \pi_{q N, \mathcal{M}_{P_{t}}}(t)\right) \\
\vdots \\
\left(X_{N, \mathcal{M}_{P_{t}}}(t), \pi_{N, \mathcal{M}_{P_{t}}}(t)\right)
\end{array}\right. \\
& \rightarrow\left\{\begin{array}{l}
\left(X_{q N, \mathcal{M}_{p_{0}(t)}}(t), \pi_{q N, \mathcal{M}_{p_{0}(t)}}(t)\right) \\
\vdots \\
\left(X_{N, \mathcal{M}_{p_{0}(t)}}(t), \pi_{q N, \mathcal{M}_{p_{0}(t)}}(t)\right)
\end{array} \rightarrow X_{t}^{(0)} \rightarrow X_{t}\right.
\end{aligned}
$$

Table 1: Graph summarizing the different steps of the algorithm.

\section{Stochastic models for the wave systems}

The various tests which we have conducted indicate that the realism of the stochastic models used to generate new wave systems and simulate the time evolution of existing wave systems is a crucial factor to retrieve good partitions. These models must be designed so as to permit an efficient exploration of the domain of the spectral parameters. In particular, the simulations should cover a domain which contains only realistic values given the situation at the previous time step and the climatology of the point of interest but which is also large enough to explore all the possible scenarios. Furthermore, the simulations should not be too much time consuming.

\section{First partition of the spectra}

In order to calibrate these stochastic models a first partition has been performed using the local wave age (ratio of wave and wind celerities) criterion. More precisely, we first compute the separation period (see [8]) $T_{s}=\frac{4}{3} \frac{2 \pi}{g} U$ where $U$ denotes the wind speed and assume that wave components with a period lower than $T_{s}$ and a direction $\theta$ such that $\cos (\theta-\Phi)>0$, where $\Phi$ denotes the wind direction, are generated 
by local winds whereas others correspond to swells. Spectral parameters $\left(H_{s}, T_{p}, \ldots\right)$ are then extracted from each part of the wave spectra. This simple method permits to retrieve two time series of spectral parameters, one for the wind seas and one for the swells. These time series have been used to estimate the parameters of the stochastic models introduced in this section.

\section{Simulation of the time evolution of the wave systems}

If $z_{t-1}$ denotes the value of one of the spectral parameters with positive values (ie $H_{s}, T_{p}, \gamma$ or $s$ ) at time $t-1$, possible values of this parameter at time $t$ were simulated according to

$$
z_{t}=s+\left|z_{t-1}-z_{\min }+e_{t}\right|
$$

where $e_{t}$ denotes a Gaussian variable with zero mean and standard deviation $\sigma_{d y n}$. The values of $z_{\text {min }}$, which correspond to the minimum value of the random variable $z_{t}$, and $\sigma_{d y n}$, which describes the temporal variability of $z_{t}$, are given in Table 3 . Generally $z_{\text {min }}$ is small compared to the typical values of $z_{t-1}$ and $\left|z_{t-1}-z_{\text {min }}+e_{t}\right|>0$. In this case Eq. 5 reduces the usual random walk model

$$
z_{t}=z_{t-1}+e_{t}
$$

which was used for the directional parameter $\theta_{m}$. Each spectral parameter is simulated independently from the others. Using more realistic models, which take into account for example that $T_{p}$ decreases with time in swell events (see e.g. Figure 5) or the strong relation between $H_{s}$ and $T_{p}$, may help improving the results obtained with the methodology introduced in this paper and this has to be further investigated (see e.g. [13]).

\section{Simulation of new swell systems}

The new swell systems are randomly drawn in the time series of swells identified using the period separation method described above. In order to allow swell systems with spectral parameters different from the ones identified using the separation method, a Gaussian noise with zeros mean and standard deviation $\sigma_{\text {new }}$ given in Table 3 is added.

\section{Simulation of new wind sea systems.}

The spectral parameters of the new wind sea systems are simulated conditionally to the wind conditions. Various simplified models have been proposed in the literature to relate the parameters $H_{s}$ and $T_{p}$ of the wind sea to the local wind speed $U$ and the fetch $F$. If the local water depth is sufficiently deep and the wind is blowing long enough, power law functions of the form

$$
H_{s}=A U^{\alpha_{U}} F^{\alpha_{F}}, T_{p}=B U^{\beta_{U}} F^{\beta_{F}}
$$

are generally used (see e.g. [9] and references therein).

Applying the log-transformation leads to linear models

$$
\begin{aligned}
\log \left(H_{s}\right) & =\log (A)+\alpha_{U} \log (U)+\alpha_{F} \log (F) \\
\log \left(T_{p}\right) & =\log (B)+\beta_{U} \log (U)+\beta_{F} \log (F)
\end{aligned}
$$

where the fetch $F$ is an unobserved variable and $\alpha_{U}, \alpha_{F}, \beta_{U}$ and $\beta_{F}$ are unknown parameters. If we model $F$ as a random variable with log-normal distribution, we get a usual Gaussian linear model which parameters can be estimated using standard procedure. It may also be interesting to include the wind direction in the model since it may be closely related to the fetch and more generally the characteristics of the wind field which generates the wave systems. In practice the wind direction $\Phi$ may be introduced as a categorical variable $\Phi_{\text {cat }}$ (we use 8 sectors of 45 degrees) or as a continuous variable (in which case we perform regression on $\cos (\Phi)$ and $\sin (\Phi))$ in the linear model.

The various models have been fitted to the data using the least-square method. According to Table 2, the best model is the most complicated one where the wind direction is included as a categorical variable and interaction between wind speed and wind direction is considered. In this model, distinct linear regression models of the form (Eq. 7-8) are fitted in each wind sector and the parameters values are shown on Figure 3. These values are coherent with the ones reported in the literature (see e.g. [9]). 


\begin{tabular}{|l|c|c|}
\hline Model & $N_{p}$ & $R_{a j}^{2}$ \\
\hline $\log \left(H_{s}\right) \sim \log (U)$ & 2 & 0.7498 \\
$\log \left(H_{s}\right) \sim \log (U)+\cos (\Phi)+\sin (\Phi)$ & 4 & 0.7784 \\
$\log \left(H_{s}\right) \sim \log (U)+\Phi_{c a t}$ & 13 & 0.7885 \\
$\log \left(H_{s}\right) \sim \log (U) * \Phi_{c a t}$ & 24 & 0.8080 \\
\hline $\log \left(T_{p}\right) \sim \log (U)$ & 2 & 0.4054 \\
$\log \left(T_{p}\right) \sim \log (U)+\cos (\Phi)+\sin (\Phi)$ & 4 & 0.4854 \\
$\log \left(T_{p}\right) \sim \log (U)+\Phi_{c a t}$ & 13 & 0.5233 \\
$\log \left(T_{p}\right) \sim \log (U) * \Phi_{c a t}$ & 24 & 0.5711 \\
\hline
\end{tabular}

Table 2: Number of parameters $N_{p}$ (second column) and adjusted $R^{2}$ coefficient (third column) for the various regression models described in the first column. The sign ' + ' corresponds to the linear model without interaction and the sign '*' to the linear model with interactions.
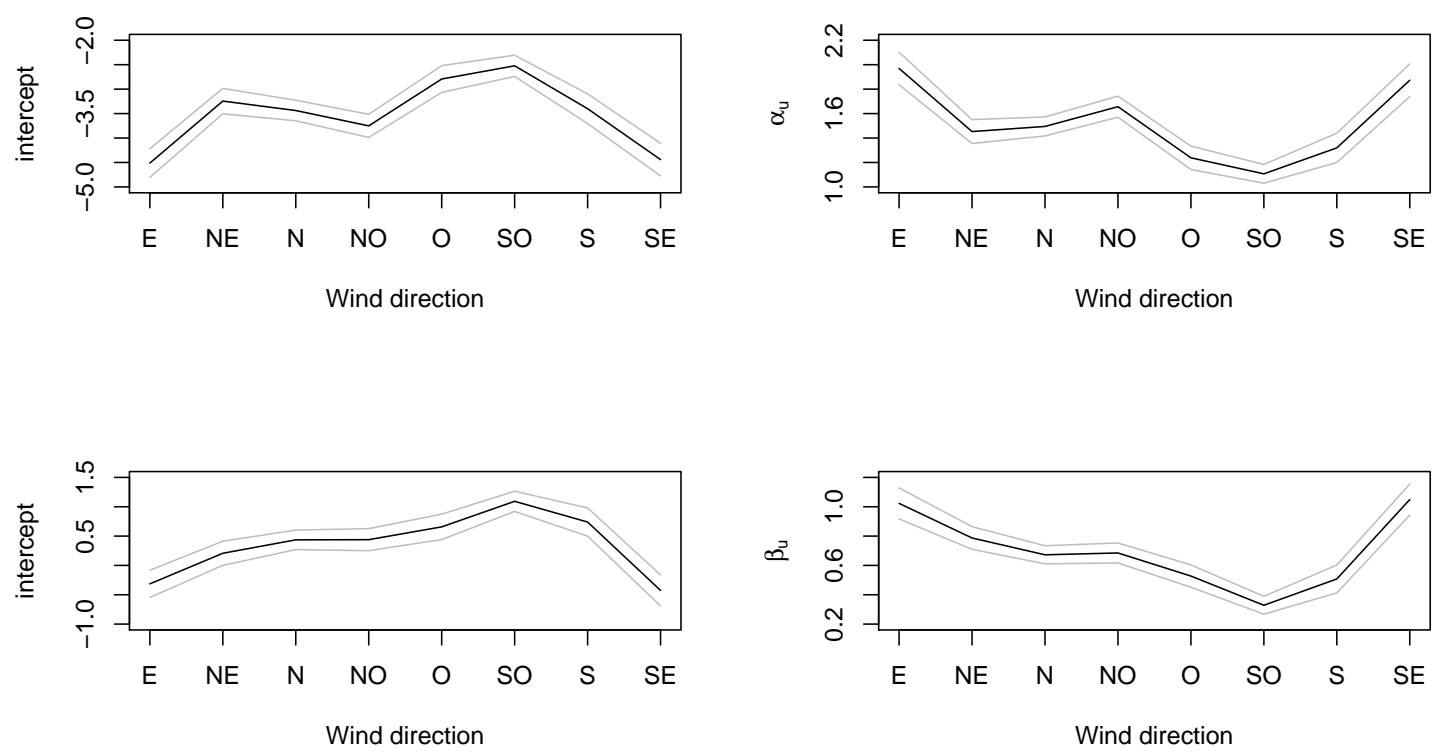

Figure 3: Evolution of the intercept (left panel) and slope (right panel) of the models Eq. (7) (top) and Eq. (8) (bottom) as a function of the wind direction (x-axis). Grey lines are $95 \%$ confidence intervals. 


\begin{tabular}{c|ccccc}
\hline & $H_{s}(m)$ & $T_{p}(s)$ & $\gamma$ & $\theta_{m}(\mathrm{rad})$ & $s$ \\
\hline$z_{\min }$ & 0 & 0 & 1 & $\mathrm{NA}$ & 1 \\
$\sigma_{\text {dyn }}$ (swell) & 0.3 & 0.3 & 0.5 & 0.1 & 1 \\
$\sigma_{\text {dyn }}$ (wind sea) & 0.3 & 0.3 & 0.7 & 0.1 & 2.5 \\
$\sigma_{\text {new }}$ (swell) & 1.5 & 1.5 & 4 & 0.3 & 15 \\
\hline Constraints & \multirow{2}{*}[0,20]{} & $\sqrt{\frac{H_{s}}{0.1}}, 20$ & {$[1,100]$} & {$[0,2 \pi]$} & {$[1,100]$} \\
\hline
\end{tabular}

Table 3: Value of the parameters used to simulate the time evolution of the spectral parameters $\left(s\right.$ and $\sigma_{d y n}$, see Section 3$)$ and the arrival of new systems $\left(\sigma_{\text {new }}\right.$, see Section 3$)$ and constraints imposed on the parameters values in the reestimation step.

\section{Numerical Results}

In this section, the algorithm introduced in the previous sections is validated on two datasets. In Section 4.1, we first consider time series of synthetic spectra in order to validate the methodology in an idealized situation where the "true" partitions are known. Then, in Section 4.2 we focus on the time series of WWIII spectra and compare the results obtained with the dynamical partitioning and SPOP algorithms.

\subsection{Simulated data}

Time series of synthetic spectra have been simulated by adding a noise to the spectra identified by applying the SPOP algorithm to the time series of WWIII spectra. More precisely, at each time step $t$, a spectrum is simulated using Eq. (1) where $X_{t}$ denotes the vector of sea state parameters identified at time $t$ using SPOP (see Figure 5 ) and $S_{\text {res }}$ is simulated from a multivariate Gaussian distribution with zero mean and covariance matrix $\Omega$ defined as

$$
\Omega_{i j}=\sigma^{2} \exp \left(-\lambda_{1}\left|\frac{1}{\omega_{i}}-\frac{1}{\omega_{j}}\right|-\lambda_{2}\left(1-\cos \left(\theta_{i}-\theta_{j}\right)\right)\right), \quad i, j=1, \cdots, n_{d} .
$$

where $\left\{\left(\omega_{i}, \theta_{i}\right)\right\}_{i=1, \cdots, n_{d}}$ denotes the frequency-direction bands into which the spectrum is discretized. In practice, we have fixed the values $\lambda_{1}=0.5$ and $\lambda_{2}=10$ but let the value of $\sigma$ vary from 0 to 0.03 (the latter case corresponds to an observation error with a $H_{s}$ of about 2.32 meters) in order to check the performance of the extraction algorithm when the noise to signal ratio increases. The terms $\lambda_{1}$ and $\lambda_{2}$ model respectively the correlation between the different frequency and direction with $\lambda_{1}=\lambda_{2}=0$ corresponding to the independent case.

The algorithm is run with $N=1000$ particles and the number of wave systems is limited to three systems with a maximum of two swells and one wind sea. This corresponds to physically realistic values for the location considered in this work. The weights Eq. (3) are computed with the covariance matrix Eq. (9) and the values $\sigma=0.1, \lambda_{1}=0.5$ and $\lambda_{2}=10$. We use a different value of $\sigma$ to generate the spectra and for the extraction algorithm since this value is generally unknown for practical applications. In the selection step (see Section 3), we consider the quantiles of order $q=0.95$ to select the best particles for each combination of sea-states. The prior distribution is defined so as to promote persisting systems and penalize the arrival of new swells when swell systems already exist. More precisely, the prior probability of a combination with at least one persisting system is 1.4 times higher than the probability of the other combinations, the prior probability of a combination with two persisting swells is 1.1 times higher and the prior probability of a combination where a new swell is added when one swell is already existing is 1.4 times smaller than the probability of the other combinations. Finally, in the reestimation step, we impose the constraints given in Table 3 on the parameters in order to avoid convergence to unrealistic values.

At each time step $t$, we obtain three different spectra: the "true" spectrum $S_{S P O P}(t)$ reconstructed using Eq. (2) and the sea state parameters obtained using the SPOP algorithm, the noisy spectrum $S_{S I M}(t)$ obtained by adding a noise to $S_{S P O P}(t)$, which has been used as input to the dynamical partition 

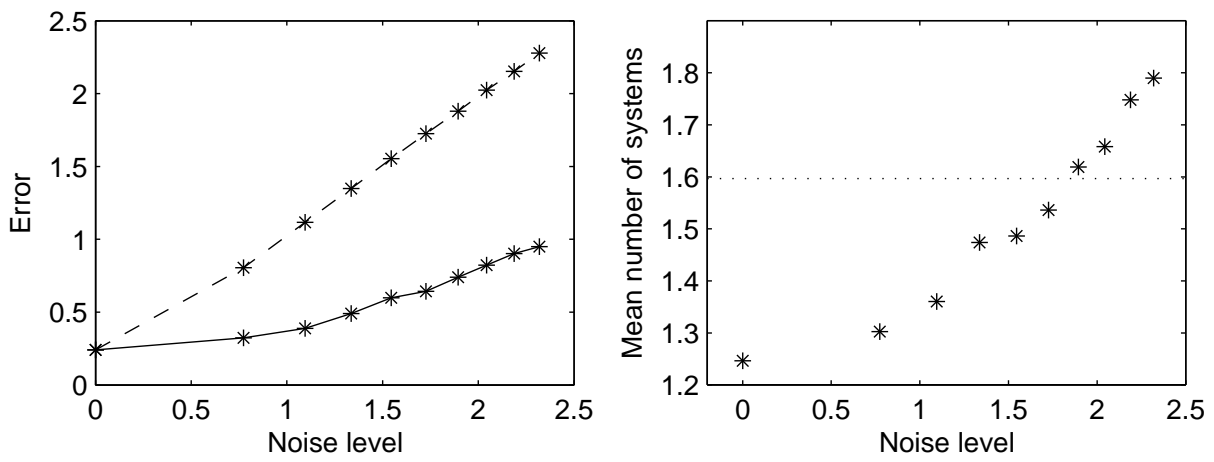

Figure 4: Left panel: median of the distances $D\left(S_{D Y N}(t), S_{S P O P}(t)\right)$ (full line) and $D\left(S_{D Y N}(t), S_{S I M}(t)\right)$ (dashed line) as a function of the median of the noise level $D\left(S_{S P O P}(t), S_{S I M}(t)\right)$ (x-axis). Right panel: mean number of systems in $S_{D Y N}(t)$ (stars) and $S_{S P O P}(t)$ (dotted line) as a function of the median of the noise level $D\left(S_{S P O P}(t), S_{S I M}(t)\right)$ (x-axis).

algorithm and the spectrum $S_{D Y N}(t)$ reconstructed using Eq. (2) and the sea state parameters identified by the dynamical partition algorithm. In order to measure the distance between these different spectra, we define

$$
D\left(S_{1}, S_{2}\right)=4 \sqrt{\int_{0}^{+\infty} \int_{0}^{2 \pi}\left|S_{1}(\omega, \theta ; t)-S_{2}(\omega, \theta ; t)\right| d \theta d \omega}
$$

where $S_{1}$ and $S_{2}$ denote two spectra. This quantity is homogeneous to a significant wave height (meters). Figure 4 shows the evolution of distances $D\left(S_{D Y N}(t), S_{S P O P}(t)\right)$ and $D\left(S_{D Y N}(t), S_{S I M}(t)\right)$ as a function of the noise level $D\left(S_{S P O P}(t), S_{S I M}(t)\right)$. It indicates that the reconstructed spectra $S_{D Y N}(t)$ are closer to the true spectra $S_{S P O P}(t)$ than to the noisy ones $S_{S I M}(t)$ and thus that the algorithm is robust to observation errors. This good behavior lets us expect that the algorithm will perform well on buoy spectra which are known to be noisy and thus difficult to analyze with classical extraction algorithms. Figure 4 also shows that our methodology tends to underestimate the number of wave systems when the noise level is low. We performed visual inspection and found that some wind sea systems with low energy are not retrieved. It generally occurs when the wind direction does not match the mean direction of the wind sea system (see Figure 5 around time 400 for example). For higher noise levels, the algorithm tends to identify too many wave systems but still identifies the main systems. The extra systems which are identified have a low energy and the values of $\gamma$ and $s$ associated with them would correspond to unrealistic spectra with an important spreading in frequency and direction; a post-treatment based on these parameters could be easily implemented to filter out the non-physical systems.

\subsection{WWIII data}

The algorithm has then been tested on the time series of WWIII spectra. In practice, we use the same parameters values than in the previous section except that the number of particles is increased to $N=10000$ in order to better explore the space of the spectral parameters and reduce the sampling variability. This leads to a significant increase of the CPU time with about 10 hours (only 15 minutes for SPOP) of computational time on a standard laptop to process three months of data. Using more realistic dynamical models would permit to largely reduce the number of particles and the computational time.

The time series of extracted sea state parameters are shown on Figure 5. These plots show periods of time with one clearly dominating swell which has a smooth temporal evolution. At other periods, two swell systems with higher temporal variability are extracted. This is further discussed below. Figure 6 shows a quantile-quantile plot of the distances between the reconstructed spectra $S_{D Y N}(t)$ and the observed WWIII spectra, denoted $S_{W W I I I}(t)$, against the distances between $S_{S P O P}(t)$ and $S_{W W I I I}(t)$. The quantiles of the dynamical partitioning method are significantly smaller than those corresponding to the SPOP method. It means that the dynamical partitioning method reproduces more accurately the time series of WWIII spectra, but it also tends to identify a higher number of systems (2.1 systems in average 

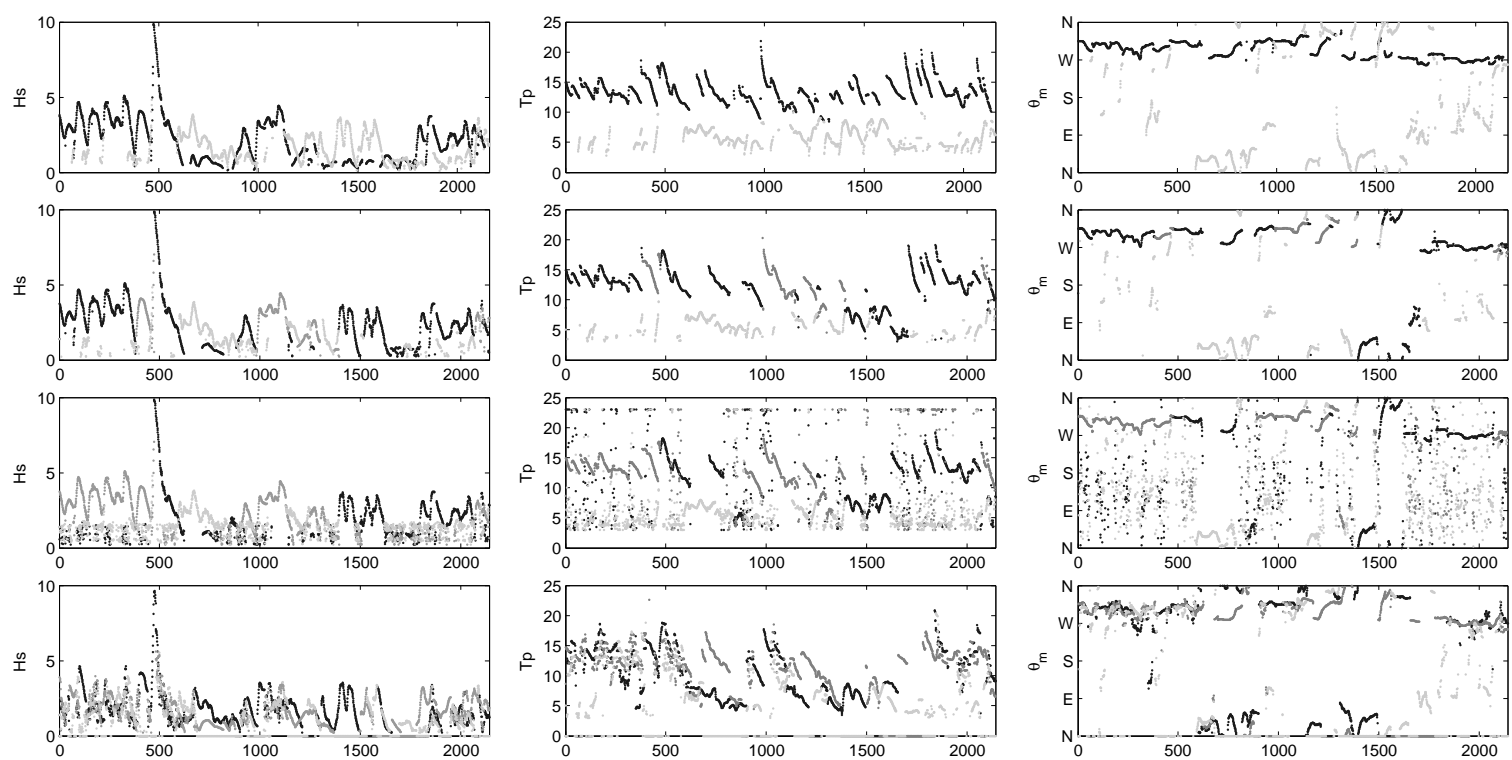

Figure 5: Time series of parameters $H_{s}, T_{p}, \theta_{m}$ and $s$ extracted using SPOP (top panels) and extracted using the dynamical partitioning method from simulated spectra time series without noise (second line), a noise with $\sigma=.03$ (third line) and from WWIII spectra (bottom panels). The black and dark gray points correspond to swell systems and the light gray to wind sea systems. The x-axis is the time in hour from the beginning of 2009.

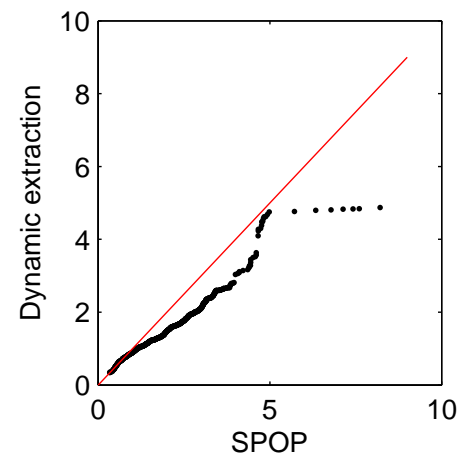

Figure 6: Quantile-quantile plot of $D\left(S_{D Y N}(t), S_{W W I I I}(t)\right)$ (y-axis) against $D\left(S_{S P O P}(t), S_{W W I I I}(t)\right)$ (x-axis).

against 1.6 for SPOP). The visual inspections we performed confirmed that the dynamical partitioning algorithm tends to over-estimate the number of systems and sometimes extracts wave systems with spectral parameters close to each other. It permits to reproduce the shapes of the WWIII spectra which are sometimes too complex to be accurately reconstructed with a single JONSWAP- $\cos ^{2} s$ spectrum. For instance, Figure 7 shows the sequence of spectra identified using the dynamical partitioning algorithm on the sequence of WWIII spectra shown on Figure 1. Three systems are identified by the algorithm at each time step of this sequence, one for the swell system which can be reasonably modeled by a single JONSWAP- $\cos ^{2 s}$ but two for the wind sea system which exhibits a more complex asymmetric shape. Using more complex parametric models for the spectra could help solving this issue. Using a posttreatment to merge the wave systems with spectral parameters close to each other (see e.g. [10]) may also lead to an improvement of the outputs of the algorithm and produce more realistic climatologies. 


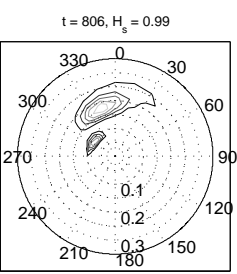

$\mathrm{t}=806, \mathrm{H}=0.97$
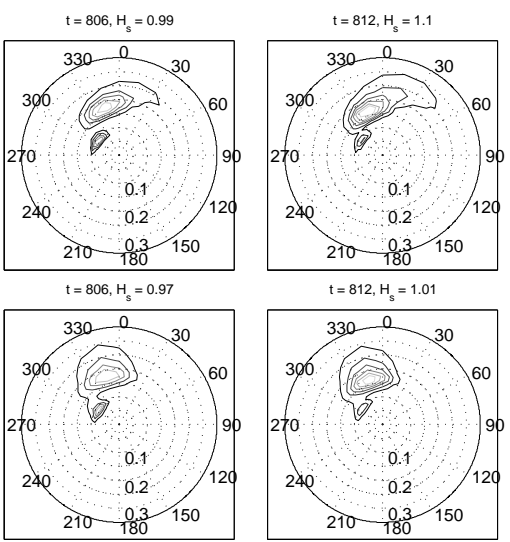

$=812, H_{s}=1.01$

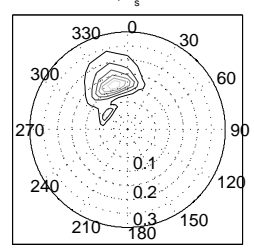

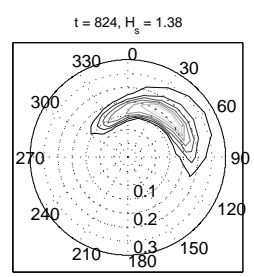

$\mathrm{t}=818, \mathrm{H}_{\mathrm{s}}=1.28$
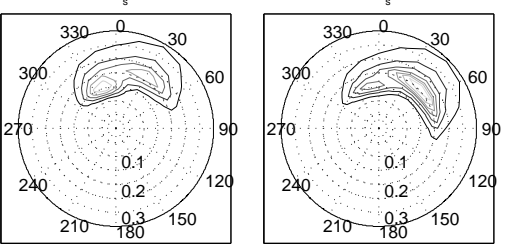

Figure 7: Example of time series of WWIII spectra (first line) and reconstructed spectra (second line). The time given in the titles corresponds to the number of hours since the beginning of 2009 .

\section{Conclusions}

A new method for partitioning directional wave spectra has been introduced. It is based on a statespace formulation which uses simple stochastic models to describe the dynamics of the wave systems and parametric models to relate the observed spectra to the synthetic parameters of the various systems. The partition obtained at a given time uses the information available at the previous time step and the method permits in particular to track automatically the time evolution of the wave systems.

The algorithm has first been validated on time series of synthetic spectra and the good results which have been obtained indicate that the model is robust to observation errors. The algorithm has then been validated on time series of WWIII spectra and the results have been compared with the ones obtained using an approach largely discussed in the literature. Again, the results are promising but the algorithm tends to identify too many systems when the shape of the spectrum is complex and can not be modeled as a mixture of JONSWAP- $\cos ^{2} s$ spectrum. We believe that this could be improved by using more flexible parametric models for the directional spectra. We have also observed that the algorithm is sensitive to the stochastic models used to describe the dynamics of the wave systems. The models which are used in this paper are very simple and refining them could also lead to substantial improvements. The methodology should now be validated on other datasets including in-situ measurements. We will also consider extending the approach in a space-time context and using the information available at other locations to build an a priori distribution. This could lead to an improvement of the partitions and also enable a tracking of the wave systems in space and time.

\section{Acknowledgments}

The authors are grateful to the three anonymous referees for their constructive comments and suggestions which led to significant improvements in the paper.

\section{References}

[1] T.W. Gerling. Partitioning sequences and arrays of directional ocean wave spectra into component wave systems. Journal of Atmospheric and Oceanic Technology, 9:444-458, 1992.

[2] A. Guillaume. Statistical tests for the comparison of surface gravity wave spectra with application to model validation. Journal of Atmospheric and Oceanic Technology, 7:552-567, 1990. 
[3] J. L. Hanson and O. M. Phillips. Automated analysis of ocean surface directional wave spectra. Journal of Atmospheric and Oceanic Technology, 18:277-293, 2001.

[4] J.L. Hanson. Wind sea growth and swell evolution in the Gulf of Alaska. PhD thesis, Johns Hopkins University, 1996.

[5] J.L. Hanson and Jensen R.E. Wave system diagnostics for numerical wave models. In 8th International Workshop on Wave Hindcasting and Forecasting, Oahu, Hawaii, 2004.

[6] S. Hasselmann, C. Brüning, K. Hasselmann, and P. Heimbach. An improved algorithm for retrieval of ocean wave spectra from synthetic aperture radar image spectra. Journal of Geophysical Research, 101:16615-29, 1996

[7] S. Hasselmann, K. Hasselmann, and C. Bruning. Dynamics and Modelling of Ocean Waves, chapter Extraction of wave spectra from SAR image spectra, pages 391-401. Cambridge University Press, 1994.

[8] P. A. Hwang, J. Ocampo-Torres, and H. Garcia-Nava. Wind sea and swell separation of 1d wave spectrum by a spectrum integration method. Journal of Atmospheric and Oceanic Technology, 29:277-293, 2012.

[9] P.A. Hwang. Duration- and fetch-limited growth functions of wind-generated waves parameterized with three different scaling wind velocities. Journal of Geophysical Research, 111, 2006.

[10] M.A. Kerbiriou, M. Prevosto, and C. Maisondieu. Influence of an improved sea-state description on a wave energy converter production. In Proceedings of 26th International Conference on Offshore Mechanics and Arctic Engineering, San Diego, 2007.

[11] J. Lefevre, C. Quentin, D. Hauser, and J. Bidlot. Measuring and analysing the directional spectrum of ocean waves, chapter Partitioning of wave spectra, pages 441-454. COST Office, 2005.

[12] W.H. Michel. Sea spectra revisited. Marine technology, 36:211-227, 1999.

[13] M. Olagnon, K. Kpogo-Nuwoklo, and Z. Guédé. Statistical processing of west africa wave directional spectra time-series into a climatology of swell events. Submitted to Journal of Marine Systems, 2013.

[14] J. Portilla and Monbaliu J. Ocampo-Torres, F.J. Spectral partitioning and identification of wind sea and swell. Journal of Atmospheric and Oceanic Technology, 26:107122, 2009.

[15] C. P. Robert and G. Casella. Monte Carlo statistical methods. Springer Texts in Statistics. Springer, 2nd edition, 2004.

[16] J.B. Saulnier, M. Prevosto, and C. Maisondieu. Refinements of sea state statistics for marine renewables: A case study from simultaneous buoy measurements in portugal. Renewal energy, 11(36):2853$65,2011$.

[17] P. Soille. Morphological Image Analysis: Principles and Applications. Springer-Verlag, 1999.

[18] L. Vincent and P. Soille. Watersheds in digital spaces: An efficient algorithm based on immersion simulations. IEEE Transactions of Pattern Analysis and Machine Intelligence, 13(6):583-598, 1991.

[19] D.W. Wang and P.A. Hwang. An operational method for separating wind sea and swell from ocean wave spectra. Journal of Atmospheric Oceanic Technology, 18:2052-2062, 2001.

[20] J. Waters, L. Wyatt, J. Wolf, A. Hines, and M. Holt. A comparison of partitioning chen applied to radar and wave spectra. In PECS 2008, pages 307-310, Liverpool, 2008. 